OPEN ACCESS

Edited by:

Jarlath E. Nally,

United States Department of

Agriculture, United States

Reviewed by: Gaurav Gupta,

NIIT University, India

Rohana P. Dassanayake,

National Animal Disease Center

(USDA ARS), United States

*Correspondence:

Maria Gomes-Solecki

mgomesso@uthsc.edu

${ }^{\dagger}$ Present address:

Mariana S. Guedes,

Department of Microbiology and

Molecular Medicine, University of

Geneva Medical School,

Geneva, Switzerland

Adeline M. Hajjar,

Cleveland Clinic, Lerner Research Institute, Cleveland, $\mathrm{OH}$, United States

Specialty section: This article was submitted to Microbial Immunology, a section of the journal

Frontiers in Immunology

Received: 15 June 2020 Accepted: 30 November 2020

Published: 15 January 2021

Citation:

Nair N, Guedes MS, Hajjar AM, Werts $C$ and Gomes-Solecki $M$ (2021) Role of TLR4 in Persistent Leptospira interrogans Infection: A Comparative In Vivo Study in Mice.

Front. Immunol. 11:572999. doi: 10.3389/fimmu.2020.572999

\section{Role of TLR4 in Persistent Leptospira interrogans Infection: A Comparative In Vivo Study in Mice}

\author{
Nisha Nair ${ }^{1}$, Mariana S. Guedes ${ }^{1 \dagger}$, Adeline M. Hajjar ${ }^{2 \dagger}$, Catherine Werts ${ }^{3,4}$ \\ and Maria Gomes-Solecki ${ }^{1 *}$ \\ ${ }^{1}$ Department of Microbiology, Immunology and Biochemistry, The University of Tennessee Health Science Center, Memphis, \\ TN, United States, ${ }^{2}$ Department of Comparative Medicine, University of Washington, Seattle, WA, United States, ${ }^{3}$ Institut \\ Pasteur, Biology and Genetics of the Bacterial Cell Wall Unit, Paris, France, ${ }^{4}$ CNRS, UMR 2001 Microbiologie intégrative et \\ Moléculaire, Paris, France
}

Toll-Like Receptor (TLR) 4, the LPS receptor, plays a central role in the control of leptospirosis and absence of TLR4 results in lethal infection in mice. Because human TLR4 does not sense the atypical leptospiral-LPS, we hypothesized that TLR4/MD-2 humanized transgenic mice (huTLR4) may be more susceptible to leptospirosis than wildtype mice, and thus may constitute a model of acute human leptospirosis. We infected huTLR4 mice, which express human TLR4 but not murine TLR4, with a high dose of $L$. interrogans serovar Copenhageni FioCruz (Leptospira) in comparison to C57BL/6J wildtype (WT) and, as a control, a congenic strain in which the t/r4 coding sequences are deleted (muTLR4 ${ }^{\text {Lps-del)}}$. We show that the huTLR4 gene is fully functional in the murine background. We found that dissemination of Leptospira in blood, shedding in urine, colonization of the kidney and overall kinetics of leptospirosis progression is equivalent between WT and huTLR4 C57BL/6J mice. Furthermore, inflammation of the kidney appeared to be subdued in huTLR4 compared to WT mice in that we observed less infiltrates of mononuclear lymphocytes, less innate immune markers and no relevant differences in fibrosis markers. Thus, huTLR4 mice showed less inflammation and kidney pathology, and are not more susceptible to leptospirosis than WT mice. This study is significant as it indicates that one intact TLR4 gene, be it mouse or human, is necessary to control acute leptospirosis.

Keywords: Leptospira, leptospirosis, humanized TLR4/MD-2 mouse model, innate immunity, kidney, TLR4

\section{INTRODUCTION}

Leptospira spp (Leptospira) are not considered gram-negative bacteria but they produce lipopolysaccharide (LPS), a potent inflammatory cell wall component that has been shown to be less inflammatory in Leptospira. Leptospiral-LPS stimulates mouse and human toll like receptor 2 (TLR2) through abundant associated lipoproteins (1). Differences of recognition of leptospiral-LPS exist between mouse and human TLR4, in that the anchor of LPS in the outer membrane of Leptospira (LipidA) activates murine, but not human TLR4 $(1,2)$. This could be due to the lack of 
phosphate groups in Leptospira's LPS lipid A (3). Escape from human TLR4 may allow for lack of immune recognition, which may favor the leptospiral infection.

Researchers working on the function of the host TLR4 in leptospirosis used immunocompetent $\mathrm{C} 57 \mathrm{BL} / 6 \mathrm{~J}$ and its respective congenic TLR4 knockouts. They found that wildtype (WT) mice expressing competent TLR4 receptors in their immune cells are resistant to lethal infection with L. interrogans whereas TLR4 knockouts are susceptible, have larger numbers of Leptospira in tissues and succumb to infection (4). Furthermore, we and others (5-8) developed mouse models of lethal and sublethal leptospirosis using $\mathrm{C} 3 \mathrm{H}-\mathrm{HeJ}$ mice that have a point mutation in the cytoplasmic domain of the tlr4 gene (9). These data strongly suggest that the competence of the TLR4 receptor affects leptospirosis outcomes.

Our hypothesis was that if human TLR4 does not sense leptospiral-LPS (1), then transgenic mice in which murine TLR4 was replaced with human TLR4 (along with its coreceptor $\mathrm{MD}-2$ ) should be more susceptible to Leptospira dissemination as compared to congenic wild-type mice expressing murine TLR4, which could result in a more robust inflammatory response and pathology in the kidney. In this study we compared susceptibility to leptospirosis in C57BL/6J wild-type (WT) and TLR4 humanized transgenic (huTLR4) mice and evaluated signs of disease progression, as well as pathology and inflammation as we previously described $(8,10)$. As a control we used a congenic mutant strain in which the tlr4 coding sequences are deleted (muTLR $4^{\text {Lps-del }}$ ), as we expected it to develop leptospirosis as shown for other C57BL/6J TLR4 ${ }^{\mathrm{KO}}$ mice (4).

\section{MATERIALS AND METHODS}

\section{Bacterial Strains}

We used Leptospira interrogans serovar Copenhageni strain Fiocruz L1-130 (Leptospira), culture passage 2 after hamster infection, originally isolated from a patient in Brazil. Leptospira was cultured as previously described (11) and enumerated by dark-field microscopy (Zeiss USA, Hawthorne, NY) that was confirmed by qPCR (StepOne Plus, Life Technologies, Grand Island, NY).

\section{Animals}

TLR4/MD-2 humanized transgenic mice (huTLR4) were developed by Dr Hajjar on a C57BL/6J TLR4/MD-2 double knock-out background (12-14). 6-week old huTLR4 male mice were transferred from Dr Hajjar laboratory at the University of Washington to the Laboratory Animal Care Unit of the University of Tennessee Health Science Center (LACUUTHSC) and quarantined for 3 weeks. C57BL/6J mice with a targeted mutation in TLR4 to produce a null (muTLR $4^{\mathrm{ko}}$ ) (12), were used to derive primary macrophages to stimulate with heatkilled Leptospira and other TLR agonists to verify the receptor function. Male 9-week old wild-type C57BL/6J (WT) mice and a congenic C57BL/6.10J mutant strain B6.B10ScN-Tlr4lps-del/ JthJ, stock \#007227 $(15,16)$, (muTLR4 $\left.4^{\text {Lps-del }}\right)$ were purchased from The Jackson Laboratories (Bar Harbor, ME) and acclimatized for one week at the pathogen-free environment in the LACU-UTHSC. The mutant strain (muTLR $4^{\text {Lps-del }}$ ) contains a spontaneous mutation that completely removes the tlr4 gene which results in absence of the TLR4 protein. Infections were done when mice reached 10 weeks of age.

\section{Ethics Statement}

This study was carried out in accordance with the Guide for the Care and Use of Laboratory Animals of the NIH. The protocols were approved by the University of Tennessee Health Science Center (UTHSC) Institutional Animal Care and Use Committee, Animal Care Protocol Application, Permits Number 16-070 and 19-062.

\section{Protocols}

Comprehensive protocols for infection of mice with pathogenic Leptospira, for monitoring clinical and molecular scores of disease and for evaluation of the host immune response were published recently (17).

\section{Infection of Mice}

Intraperitoneal infection was done as described previously using a dose of $\sim 10^{8}$ virulent Leptospira in sterile PBS. Bacteria were counted in a Petroff-Hausser chamber under a dark field microscope and confirmed by qPCR. Body weights were monitored daily. Urine was also collected on a daily basis for 15 days p.i. by gently massaging the bladder area and the urine was collected into sterile aluminum foil. Blood (up to 20 $\mu \mathrm{l})$ was collected every other day by tail nick for 15 days. At termination, kidneys were collected for qPCR.

\section{RT-PCR and q-PCR}

DNA was extracted per manufacturers' instructions from urine, blood, and kidney using a NucleoSpin tissue kit (Clontech). Quantification of Leptospira 16s rRNA was done using TAMRA probe and primers from Eurofins (Huntsville, AL) by real-time PCR (qPCR) (StepOne Plus). RNeasy mini kit (Qiagen) was used to extract total RNA followed by reverse transcription using a high-capacity cDNA reverse transcription kit (Applied Biosystems). Real-time PCR on the cDNA was performed as described (8). For RT-PCR, we used TAMRA probes specific for inducible nitric oxide synthase (iNOS), Collagen A1 (ColA1), keratinocyte-derived chemokine (KC, CxCL1), macrophage inflammatory protein 2 (MIP-2, CxCL2), RANTES (CCL5), tumor necrosis factor alpha (TNF- $\alpha$ ), interferon gamma (IFN$\gamma$ ) and IL-10. $\beta$-actin was used as control for the comparative CT method (10).

\section{Histopathology}

The kidney tissues were fixed in 10\% neutral buffered formalin and submitted to the UTHSC Research Histology Core Facility for histology processing. Paraffin embedded tissues were sectioned and stained with hematoxylin and eosin (H\&E). The slides were analyzed for nephritis and imaged under an Axio Zeiss Imager A1 light microscope with Axiocam 103 and Zen 2 lite software. 


\section{Profiling Immune Cell Populations by Flow Cytometry \\ Cell Preparation}

Spleens were harvested from mice, placed in RPMI 1640 (Mediatech, Manassas, VA), and diced with frozen slides in Hanks balanced salt solution medium (Cellgro, Manassas, VA) to produce cell suspensions. Red blood cell lysis was performed using $2 \mathrm{ml} /$ spleen of 1X ACK lysing buffer (Life Technologies). Cells were then washed in RPMI 1640 containing 10\% heat-inactivated fetal bovine serum (Atlanta Biotech) and 0.09\% sodium azide (SigmaAldrich, St. Louis, MO), followed by passage through $70-\mu \mathrm{m}$-poresize and 40- $\mu \mathrm{m}$-pore-size nylon filters (BD Falcon, Bedford, MA), and the cells were counted using a Luna-FL automated cell counter (Logos Biosystems, Gyunggi-Do, South Korea).

\section{Flow Cytometric Cell Staining}

Cell viability was analyzed by mixing $18 \mu \mathrm{l}$ of splenocytes with $2 \mu \mathrm{l}$ of acridine orange-propidium iodide staining solution in a Luna-FL automated cell counter, and $3 \times 10^{6}$ cells per tube were stained as follows: cells were incubated in $0.5 \mu \mathrm{g}$ Fc block (BD Biosciences) for $15 \mathrm{~min}$ at $4^{\circ} \mathrm{C}$ in staining buffer $\left(\mathrm{Ca}^{2+}-\right.$ and $\mathrm{Mg}^{2+}$ - free PBS containing 3\% heat-inactivated fetal bovine serum, 0.09\% sodium azide [Sigma-Aldrich, St. Louis, MO], 5mM EDTA), washed twice, and incubated with the appropriate marker for surface staining in the dark for $30 \mathrm{~min}$ at $4^{\circ} \mathrm{C}$. T lymphocyte staining was performed using $\mathrm{CD} 3$ clone 17A2 conjugated with fluorescein isothiocyanate (FITC) (1:200; BD Biosciences), CD4 clone RM4-5 conjugated with phycoerythrin (PE) (1:150; BD Biosciences), CD8 clone 53-6.7 conjugated with allophycocyanin (APC)-Cy7 (1:150; BD Biosciences), CD62L clone MEL-14 conjugated with PE-Cy7 (1:150; BD Biosciences), CD44 clone IM7 conjugated with APC (1:150; BD Biosciences). Flow cytometric staining and analysis was done as described (8) and acquired on ZE5 cell analyzer (Bio-Rad, Hercules, CA, USA). Analysis was performed using Flow Jo software.

\section{Splenic Macrophages}

Heat-killed Leptospira were prepared as follows: pellets from a culture, which were washed with $1 \mathrm{ml}$ PBS, were harvested by centrifugation at $12,000 \mathrm{~g}$ and heat killed by exposure to heat for $10 \mathrm{~min}$ at $95^{\circ} \mathrm{C}$. The supernatant was discarded, and the extract resuspended in endotoxin free PBS. Lack of motility was confirmed under a dark field microscope. This process was repeated to ensure no motile Leptospira were visualized.

Single-cell splenocyte suspensions were stimulated with 10 $\mu \mathrm{g} / \mathrm{ml} \mathrm{CpG} 1826$ or Pam3CSK4, $10 \mathrm{ng} / \mathrm{ml}$ and $100 \mathrm{ng} / \mathrm{ml}$ E. coli O111:B4 LPS, or $10^{7} / \mathrm{ml}$ and $10^{8} / \mathrm{ml}$ heat-killed P2 L. interrogans in the presence of $10 \mu \mathrm{g} / \mathrm{ml}$ brefeldin A for $\sim 4 \mathrm{~h}$ as described (13). Cells were then stained and analyzed using flow cytometry as described (13). CpG, E. coli (EC) LPS and Pam3cys were used as TLR9, TLR4 and TLR2 agonists, respectively.

\section{Statistics}

Two-tailed unpaired parametric t-test with Welch's correction was used to analyze differences between infected and uninfected groups. Differences between infected mice was analyzed by
Ordinary One-Way ANOVA. For immune markers and T cell populations, Ordinary One-Way ANOVA was done if at least two of the mouse strains showed significant differences between infected and uninfected mice to ensure the effect seen was due to infection. Statistical analysis was done using GraphPad Prism software, $\alpha=0.05$.

\section{RESULTS}

To find out if replacing the murine TLR4 with human TLR4 affects the kinetics of leptospirosis progression, we compared infection in wild-type C57BL/6J (WT) and humanized TLR4/ MD2 transgenic mice (huTLR4) and added a congenic murine mutant lacking the TLR4 receptor as control (muTLR $4^{\text {Lps-del }}$ ). We inoculated mice intraperitoneally with a high dose $\left(\sim 10^{8}\right)$ of L. interrogans Copenhageni FioCruz and analyzed weight loss as a clinical outcome of disease as well as Leptospira dissemination and colonization of the kidney (Figures $\mathbf{1}$ and 2). In contrast to PBS controls, both WT and huTLR4 slowly lost small but significant amounts of weight over two weeks of infection (Figures 1A, B). The muTLR $4^{\text {Lps-del }}$ lost an abrupt and considerably higher amount of weight after day 6 when colonization of the kidney takes place (Figure 1C). Differences in weight loss between the three infected groups were significant with huTLR4 being the group that lost less weight (Figure 1D).

When we quantified the burden of Leptospira in fluids and tissues by qPCR we found low numbers of Leptospira in blood in WT and huTLR4 mice (mean 230 and $~ 300$, respectively) and, as expected, a significantly higher number of Leptospira in muTLR4 Lps-del (mean 1200) (Figure 2A). Furthermore, much higher numbers of Leptospira ( 2-4 Log) were excreted in urine of all infected mice, $\sim 41,000$ in WT, 74,000 in huTLR4 and $\sim 305,000$ in muTLR $4^{\text {Lps-del }}$ (Figure 2B). At termination at $15 \mathrm{dpi}$, tissue PCR showed the same trend with a significantly higher presence of Leptospira in the kidney of muTLR $4^{\text {Lps-del }}$ (Figure 2C), and culture of the tissue showed that live Leptospira colonized the kidney of all groups of infected mice (Figure 2D). These results suggest that Leptospira infection leads to persistent sublethal leptospirosis, renal colonization and stable shedding of Leptospira in urine of WT and huTLR4 mice.

To find out if replacing murine TLR4 with human TLR4 affects inflammation of the kidney after infection with $L$. interrogans, we analyzed the differences in histopathology and inflammatory markers in tissue collected from infected and uninfected mice two weeks post infection (Figures 3 and 4). Examination of H\&E sections of kidney showed that of all three groups of infected mice, huTLR4 had considerably less mononuclear lymphocyte infiltrates and less tubular damage than WT and muTLR4 ${ }^{\text {Lps-del }}$ (Figure 3A). We purified mRNA from infected and uninfected kidney. Quantification of expression of iNOS and fibroblast activation marker collagen 1 A1 (ColA1) showed an overall lower detection of iNOS in huTLR4 as well as lower ColA1 in WT and huTLR4 than in muTLR $4^{\text {Lps-del }}$. Differences between infected groups were statistically significant for ColA1 (Figure 3B). 

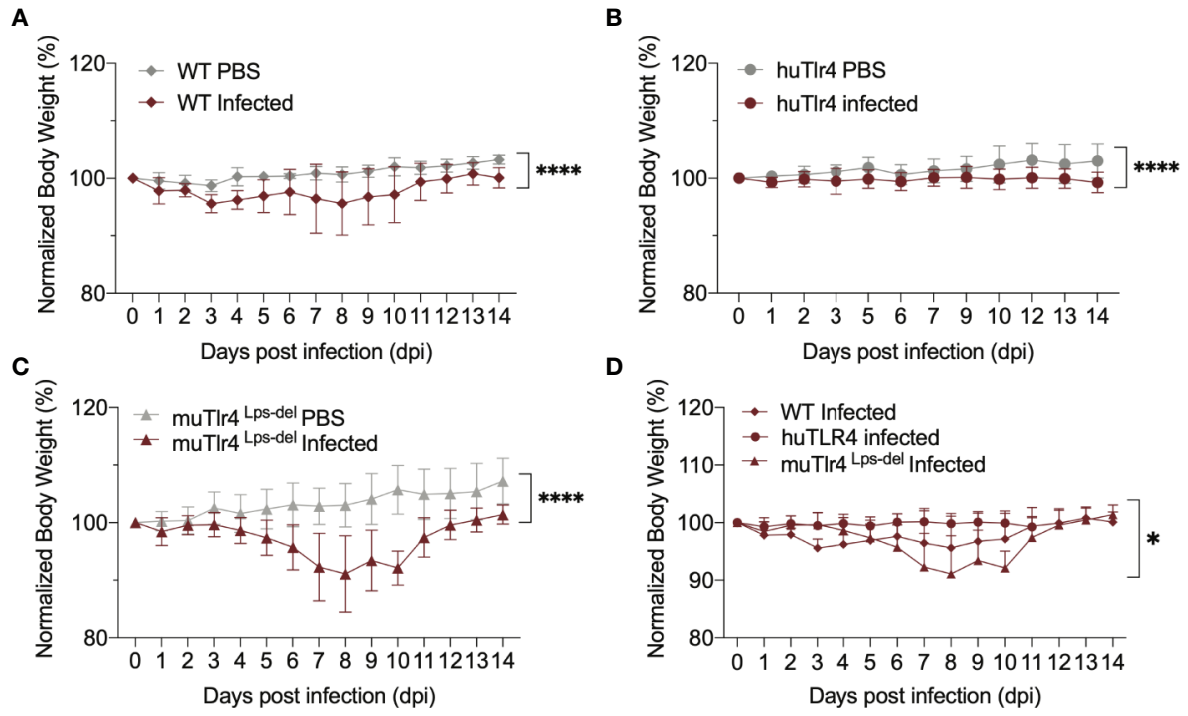

FIGURE 1 | Both wild-type (WT) and TLR4 humanized transgenic (huTLR4) mice lose small but significant weight after infection with L. interrogans Copenhageni FioCruz. Weight measurement of C57BL/6 mice carrying wild-type murine TLR4 - WT (A), human TLR4/MD2 - huTLR4 (B), and no murine TLR4 - muTLR4 ${ }^{\text {Lss-del }}$ which was used as a control (C). Mice were injected with Leptospira (Infected) or with sterile PBS (PBS), $n=7-9$ per group. Statistics A, B, C by unpaired t test with Welch's correction, ${ }^{* \star \star *} \mathrm{p}<0.0001$ and $(\mathbf{D})$, Ordinary One-way ANOVA ${ }^{*} \mathrm{p}=0.0168$. Data represents two independent experiments.

Given that these results suggest that inflammation may not be triggered in kidney of huTLR4 mice after Leptospira infection, we quantified a number of chemokines and cytokines in this tissue (Figure 4). Between infected and uninfected mice, the expression of CxCL1/KC, CxCL2/MIP-2, CCL5/RANTES, TNF- $\alpha$, IFN- $\gamma$; and IL-10 were generally significantly increased in the infected kidneys of WT, huTLR4 and muTLR ${ }^{\text {Lps-del }}$ control, with the notable exception of CxCL1/KC chemokine and IL-10 in huTLR4 (Figure 4). Between the three groups of infected mice, differences between CxCL1/KC, CxCL2/MIP2, TNF- $\alpha$ and IL-10 were significant. These results suggest that inflammation in kidneys of huTLR4 mice is reduced compared to WT and muTLR $4^{\text {Lps-del }}$ control.

We evaluated systemic splenic cellular immune responses in Leptospira infected and noninfected WT, huTLR4, and muTLR4 ${ }^{\text {Lps-del }}$ mice (Figures 5 and 6). Absolute numbers of splenocytes, CD3+, $\mathrm{CD} 3+\mathrm{CD} 4+$, and $\mathrm{CD} 3+\mathrm{CD} 8+\mathrm{T}$ cells were quantified (Figure 5). We found that, although not significant, absolute numbers of splenocytes were increased between infected and uninfected groups. Furthermore, between infected groups, absolute numbers of $\mathrm{CD} 3+, \mathrm{CD} 3+\mathrm{CD} 4+$, and $\mathrm{CD} 3+\mathrm{CD} 8+\mathrm{T}$ cells were reduced in huTLR4 in comparison to WT and muTLR $4^{\text {Lps-del }}$ mice.
A

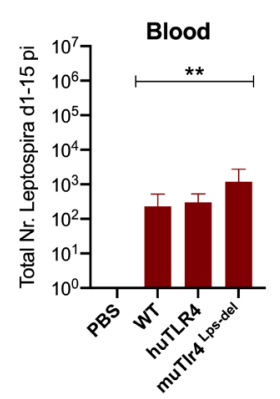

B

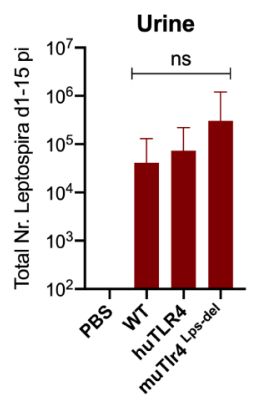

C

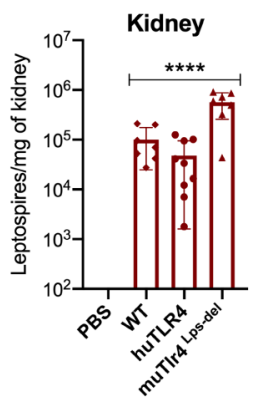

D

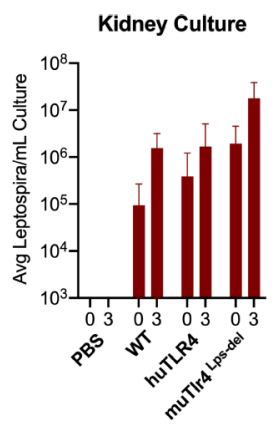

FIGURE 2 | Leptospira disseminates in blood and colonizes the kidney of wild-type (WT) and TLR4 humanized transgenic (huTLR4) mice. Quantification of total Leptospira in blood (A), urine (B), kidney tissue (C), and in kidney culture (D) by qPCR (16S rRNA). Total number (Nr) of Leptospira in blood and urine are represented as the sum of Leptospira detected between days 1-5 and days 7-14 post-infection, respectively (A, B); kidney is represented as number of Leptospira per $\mathrm{mg}$ of kidney tissue (C); kidney culture is represented by the average number of Leptospira per $\mathrm{ml}$ of culture at termination (0) and 3 days later (3) (D). Statistics by one-way ANOVA ${ }^{* \star} \mathrm{p}=0.0074,{ }^{\star \star \star \star} \mathrm{p}<0.0001$ and $\mathrm{ns}$, non-significant. Data represents two independent experiments. 
A

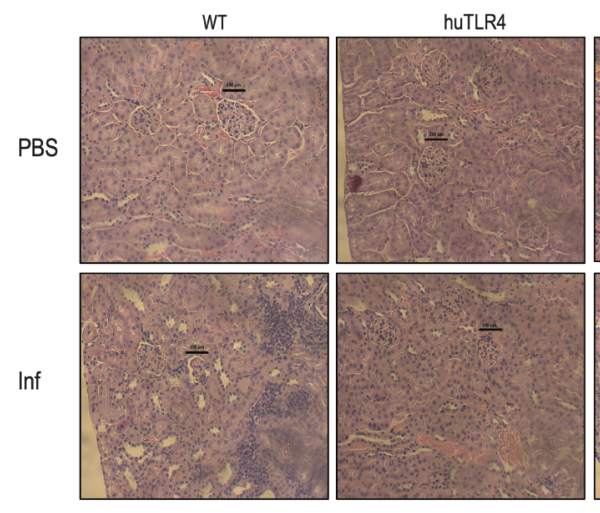

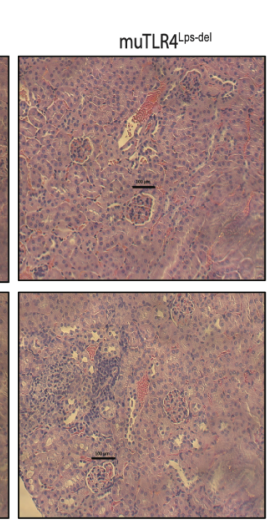

B

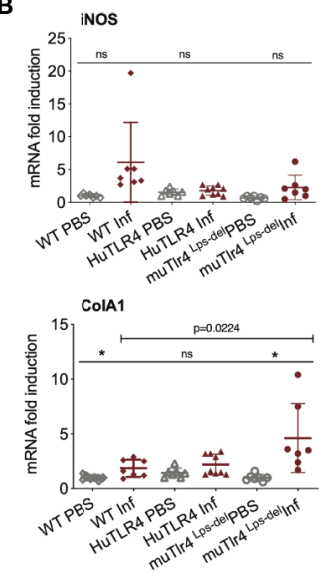

FIGURE 3 | Histopathology and measurements of antimicrobial and collagen markers in the kidney shows less immune cell engagement in TLR4 humanized transgenic (huTLR4) mice. (A) Hematoxylin and eosin (H\&E) staining (20X) of kidney sections of L. interrogans infected (Inf) and uninfected (PBS) C57BL/6J mice

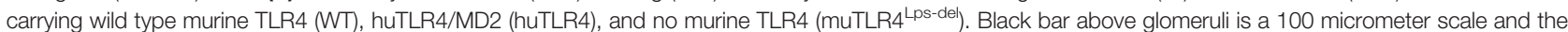
blue staining highlights infiltration of mononuclear lymphocytes. (B) qPCR quantification of antimicrobial transcripts (iNOS) and collagen marker (ColA1). Statistics by unpaired t test with Welch's correction between infected versus non-infected groups, ${ }^{*} \mathrm{p}<0.05$ and ns, non-significant, and by Ordinary One-Way ANOVA between infected groups for ColA1. H\&E data represents one of two experiments and qPCR data represents two independent experiments.

Further flow cytometric analysis of proportions of splenic T cells from WT, huTLR4 and muTLR $4^{\text {Lps-del }}$ mice infected with L. interrogans (Figure 6) showed no differences in proportions of total $\mathrm{CD} 3+, \mathrm{CD} 3+\mathrm{CD} 4+$, and $\mathrm{CD} 3+\mathrm{CD} 8+\mathrm{T}$ cells between uninfected (PBS) and infected (Inf) mice except for a significantly increased population of $\mathrm{CD} 3+\mathrm{CD} 4+$ and a significantly decreased population of CD3+CD8+ in huTLR4 mice (Figures 6A-C). CD4+ naïve T cell populations decreased
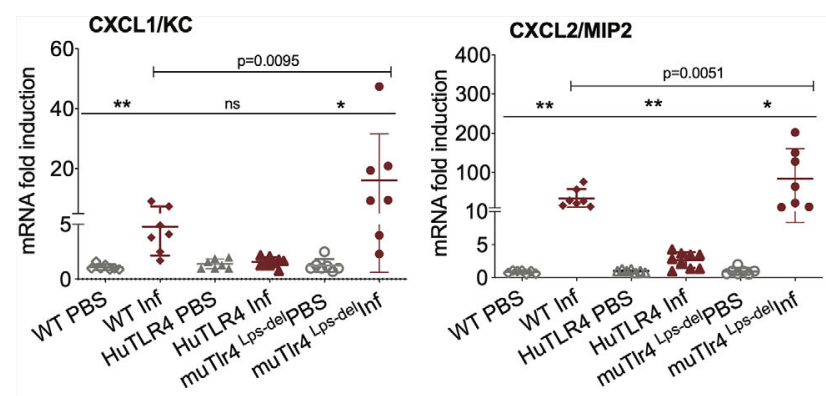

IFN- $\gamma$
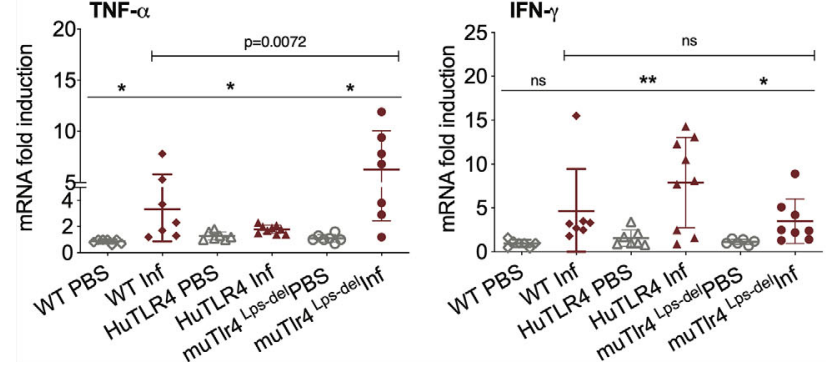

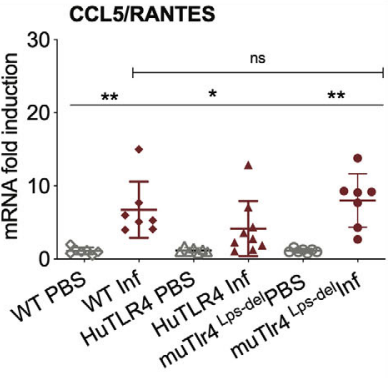

IL-10

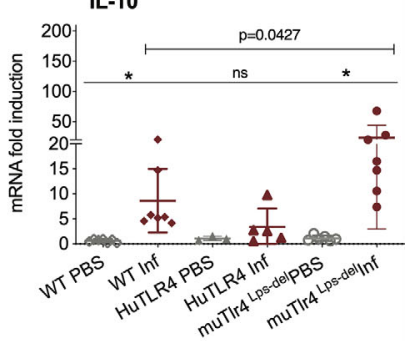

FIGURE 4 | Differences in immune markers in kidney from infected wild-type (WT) and TLR4 humanized transgenic (huTLR4) mice suggest less involvement of innate immune processes in huTLR4. qPCR quantification of reverse-transcribed pro-inflammatory innate response chemokines (CxCL1, CxCL2, CCL5) and cytokines (TNF- $\alpha$, IFN $\gamma$, IL-10) in the kidney of wildtype murine TLR4, humanized TLR4 (huTLR4) and muTLR4 ${ }^{\text {Lps-del }}$ control two weeks post-infection with L. interrogans serovar Copenhageni FioCruz. $n=7$ to 9 mice per group except IL10, $n=3$ to 7 mice per group. Statistics by unpaired t test with Welch's correction for infected versus uninfected, ${ }^{\star} \mathrm{p}<0.05,{ }^{\star *} \mathrm{p}<0.005$, and by Ordinary One-Way ANOVA between infected groups, ns, nonsignificant. Data represents two independent experiments. 


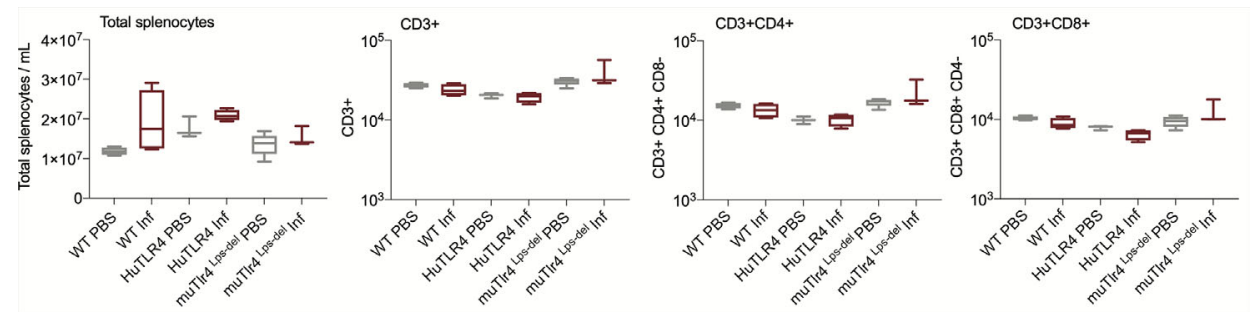

FIGURE 5 | Absolute numbers of total CD3+, CD3+CD4+, and CD3+CD8+ T cells in wild-type (WT), TLR4 humanized transgenic (huTLR4), and muTLR4 ${ }^{\text {Lps-del }}$ infected with $L$. interrogans. Splenocytes were prepared and total cells were counted on a LUNA cell counter. Cells were stained for flow cytometric analysis of $T$ lymphocyte profiles and a total of 100,000 events were captured in a ZE5 cell analyzer (Bio-Rad, Hercules, CA, USA). CD3, CD4, CD8 cell counts were obtained from single cell populations. Data are the mean \pm SD of cell counts for three or four mice per group. Statistics by Unpaired t test with Welch's correction were not significant between any of the uninfected and infected groups. Data for huTLR4 represents one of two experiments and data for WT/muTLR4 ${ }^{\text {Lps-del }}$ represents one experiment.

A

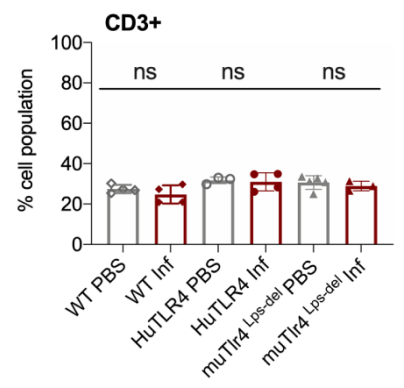

D

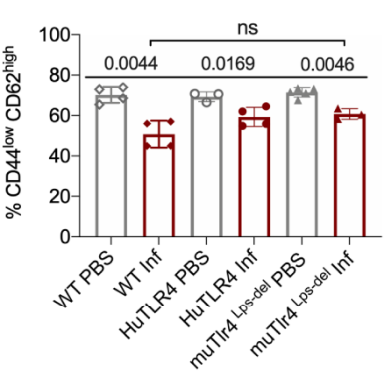

G

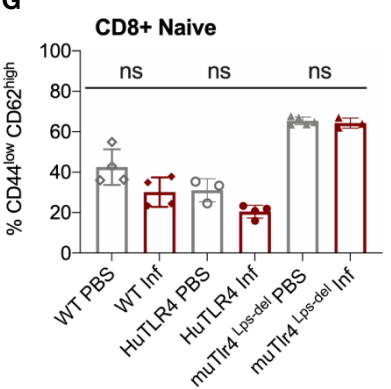

B

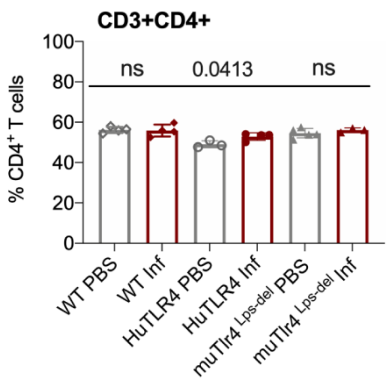

E

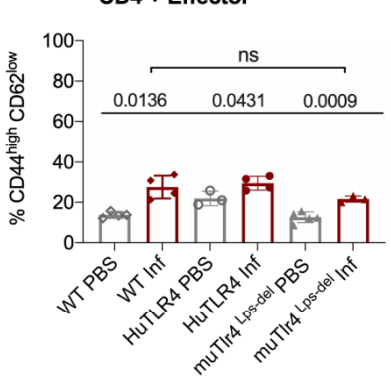

"

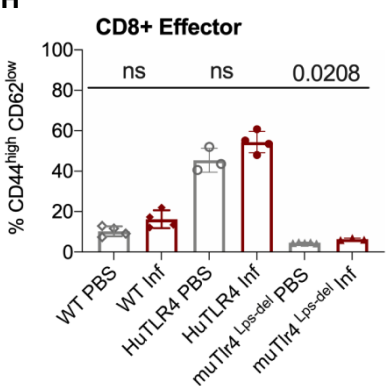

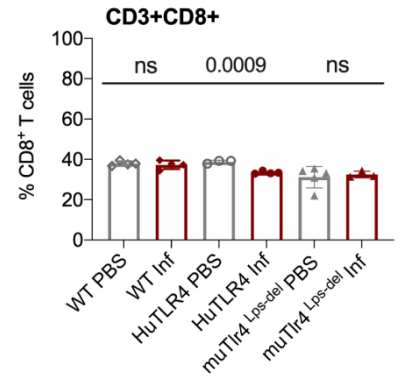

F

CD4+ Memory
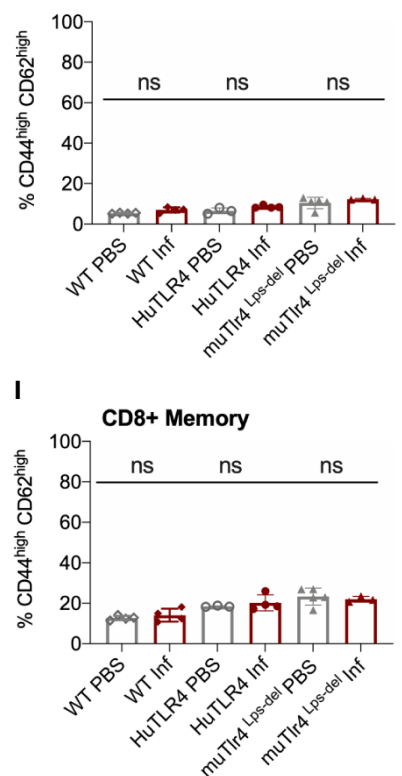

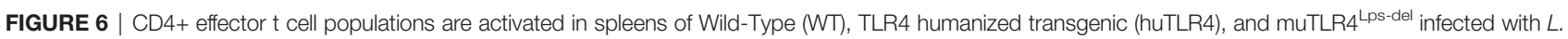
interrogans. Freshly isolated splenocytes from WT, huTLR4, and muTLR4 ${ }^{\text {Lps-del }}$ were blocked with anti-mouse CD16/CD32, incubated with the respective surface lineage markers (A-I) and acquired in a ZE5 cell analyzer (Bio-Rad, Hercules, CA, USA). Data are the mean \pm SD percentage of cells for 3 or 4 mice per group. Statistics by Unpaired t test with Welch's correction between uninfected and infected groups, and by Ordinary One-Way ANOVA between infected groups for CD4+ Naïve and CD4+ Effector; ns, non-significant. Data for huTLR4 represents one of two experiments and data for WT/muTLR4 Lps-del represents one experiment. 
between all noninfected and infected groups (Figure 6D) as they differentiated into effector T cells (Figure 6E) but not memory $\mathrm{T}$ cells (Figure 6F). Thus, as expected, naïve CD4+ $\mathrm{T}$ cells differentiated info effector T cells in infected WT, huTLR4 and muTLR $4^{\text {Lps-del }}$ mice. Proportions of CD8+ naïve T cells were slightly lower in infected than in noninfected groups (Figure 6G) as they differentiated into CD8+ effector T cells but not memory $\mathrm{T}$ cells. Differences in CD8+ effector T cells were not significant except for muTLR4 ${ }^{\text {Lps-del }}$ (Figures $6 \mathbf{H}, \mathbf{I}$ ).

To get further insight into the role of TLR4 in Leptospira induced inflammation, we compared primary splenocytes from WT and huTLR4 mice, as well as mice with a targeted knock-out mutation in TLR4 (muTLR4 ${ }^{\mathrm{KO}}$ ) (12). The cells were stimulated with $10^{7} / \mathrm{ml}$ and $10^{8} / \mathrm{ml}$ heat-killed L. interrogans and gated on the $\mathrm{CD} 11 \mathrm{~b}^{+}$macrophage/monocyte population using an ex vivo flow-cytometry based assay (13) (Figure 7). We found that 6\% and $20 \%$ of CD $11 \mathrm{~b}+$ macrophages from huTLR 4 mice stimulated with $10^{7}$ and $10^{8}$ Leptospira, respectively, produced TNF- $\alpha$ in comparison to $14 \%$ and $28 \%$ from WT, and $~ 3 \%$ and $12 \%$ from muTLR ${ }^{\mathrm{KO}}$. In addition, macrophages from all mouse genotypes responded to the same extent to $\mathrm{CpG}$ and to Pam3CSK4 (TLR9 and TLR2 agonists). WT and huTLR4 macrophages responded to LPS from E. coli (EC LPS, TLR4 agonist) in contrast to muTLR $4^{\mathrm{KO}}$ macrophages that did not produce TNF- $\alpha$. It is important to note that stimulation of WT and huTLR4 macrophages with increasing concentrations of the TLR4 agonist (10ng/ml and $100 \mathrm{ng} / \mathrm{ml}$ of EC LPS) led to the production of equivalent amounts of TNF- $\alpha$. This indicates that the huTLR4 receptor is as functional as the WT, in the murine background. Interestingly, previous quantification of relative TLR4 mRNA expression in bone marrow derived macrophages from WT and huTLR4 mice, using the same primers, showed a significantly higher expression of huTLR4 (18). A lower overall production of TNF- $\alpha$ by huTLR 4 macrophages stimulated with $10^{7}$ and $10^{8}$ heat-killed Leptospira suggests that the systemic inflammatory response of huTLR4 is reduced compared to WT mice.

\section{DISCUSSION}

The major goal of this study was to understand how TLR4 affects Leptospira infection in vivo. Another goal was to develop an immunocompetent mouse model of leptospirosis. The rationale for using humanized TLR4 transgenic mice is that, if human TLR4 does not sense the atypical leptospiral-LPS $(1,2,19)$, these mice should be more permissive of Leptospira escape and more susceptible to leptospirosis than wild-type mice expressing murine TLR4 that do sense leptospiral-LPS. We therefore hypothesized that C57BL/6J mice carrying the human TLR4/MD-2 receptor and coreceptor in its immune cells (huTLR4) should be more sensitive to sublethal leptospirosis than wild-type congenic C57BL/6J (WT). However, we found that dissemination of Leptospira interrogans serovar Copenhageni FioCruz in blood, shedding in urine, colonization of the kidney and overall kinetics of leptospirosis progression is equivalent between mice carrying murine and human TLR4. Furthermore, the inflammatory response to infection with Leptospira seemed to be subdued in huTLR4 compared to WT mice in that we observed less infiltrates of mononuclear lymphocytes, less innate immune markers and no relevant differences in fibrosis markers in kidney as well as less production of TNF $\alpha$ by murine splenic macrophages harboring a fully functional huTLR4 receptor. These results are consistent with the lack of leptospiral-LPS stimulation of human TLR4 (1).

Decreased inflammation in huTLR4 mice could have led to increased numbers of Leptospira in kidney. Surprisingly, this was not observed. We analyzed the kinetics of leptospirosis infection and colonization of the kidney between infected and uninfected humanized TLR4 mice as established previously in our laboratory $(8,10,20)$ (Figures 1 and 2). Compared to WT and

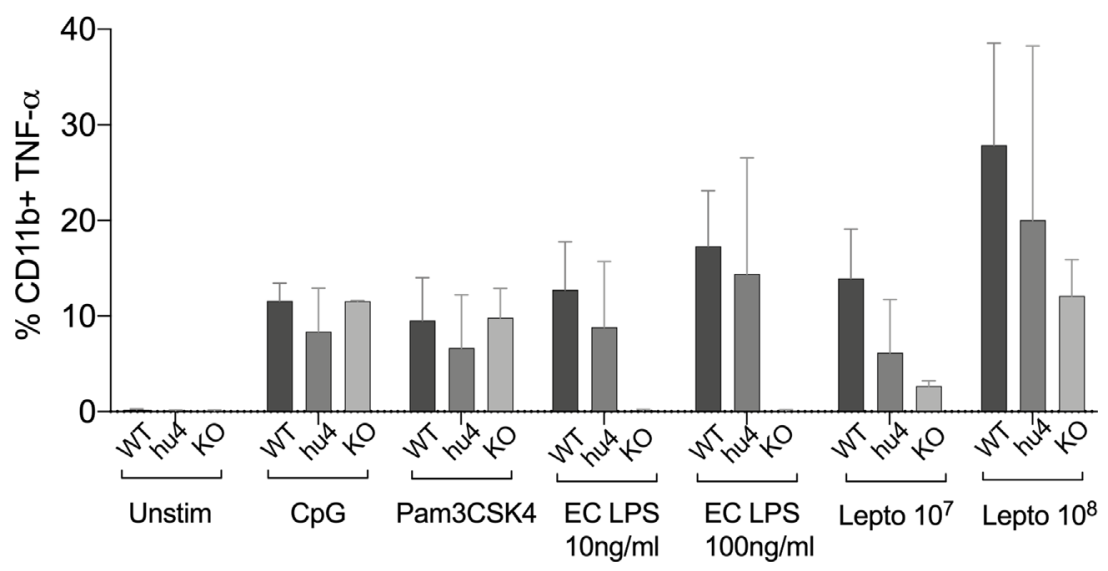

FIGURE 7 | Splenic macrophages of TLR4 humanized transgenic (huTLR4) mice produce less tumor necrosis factor alpha (TNF- $\alpha$ ) than wild-type (WT) upon Leptospira stimulation. Flow cytometric quantification of \% of primary C57BL/6J mouse monocyte/macrophages from WT, huTLR4, and muTLR4 KO producing TNF- $\alpha$ ( \pm SD) after stimulation with heat killed L. interrogans ser. Copenhageni FioCruz (Passage 2) and TLR agonists (CpG TLR9, Pam3CSK4 TLR2 and E. coli (EC) LPS TLR4). Data represents two experiments. 
to muTLR $4^{\text {Lps-del }}$, infected huTLR4 mice showed a similar pattern of low and slow loss of weight as the WT which was considerably different than the abrupt loss of weight shown by muTLR $4^{\text {Lps-del }}$ at days $\sim 6-7$ post-infection when colonization of the kidney takes place, as we previously observed in $\mathrm{C} 3 \mathrm{H}-\mathrm{HeJ}$ mice $(8,10,11,20)$. The latter carries a point mutation in the toll like receptor 4 (tlr 4 ) cytoplasmic domain that results in a lack of signaling despite presence of the receptor and binding of bacterial LPS (9). Furthermore, dissemination in blood and shedding of Leptospira in urine of WT and huTLR4 mice were equivalent as was the amount of live Leptospira detected in kidney. This again contrasted with the amount of Leptospira detected in blood, urine and kidney of muTLR ${ }^{\text {Lps-del }}$ mice that were 1 Log higher than the WT and huTLR4 counterparts. An even higher load of Leptospira was observed in blood and urine of infected C3H-HeJ mice $(8,20)$. Furthermore, wild-type C57BL/6J mice were found to have 2Log lower numbers of Leptospira in urine than TLR4 ${ }^{\mathrm{KO}}$ mice $(4,21)$. Interestingly, the results of Leptospira infection obtained in $\mathrm{C} 3 \mathrm{H}-\mathrm{HeJ}$ (containing a point mutation in the TLR4 receptor) and in C57BL/6J TLR4 ${ }^{\mathrm{KO}}$ (do not express the TLR4 receptor) are consistent with the results obtained here with $\mathrm{C} 57 \mathrm{BL} /$ 6.10J TLR4 ${ }^{\text {Lps-del }}$ which contain a spontaneous mutation that completely removes the $t l r 4$ gene and thus results in the absence of the TLR4 receptor. These results confirm that mutations resulting in a loss of response to leptospiral-LPS, either resulting from a point mutation in tlr4 in $\mathrm{C} 3 \mathrm{H}-\mathrm{HeJ}$ or from a complete deletion of the gene in C57BL6/J mice, increase susceptibility to Leptospira infection regardless of genetic background. However, this was not replicated by substitution of wildtype murine TLR4 with human TLR4, that is known to lack leptospiral-LPS sensing.

Analysis of markers of inflammation and other immune cell engagement (Figures 3 and 4) in kidney showed that huTLR4 mice exhibit less markers of innate immune cell activity (CxCL1/ KC, CxCL2/MIP-2 and TNF- $\alpha$ ) and less anti-inflammatory cytokine IL-10 than WT mice. Differences in antimicrobial iNOS and fibroblast activation marker collagen 1 A1 (ColA1) which are indicative of interstitial collagen deposition (fibrosis) were not significant between huTLR4 and WT mice. This may be further corroborated by the lower number of mononuclear lymphocyte infiltrates observed in $\mathrm{H} \& \mathrm{E}$ stained slides of the kidney. These data suggest that decreased inflammatory responses in the huTLR4 mouse kidney is dependent on TLR4. If human TLR4 does not sense the atypical Leptospira LPS (1, 2, 19), huTLR4 mice should have been more permissive of Leptospira escape and more susceptible to leptospirosis than wild-type. However, the opposite was observed. The data could suggest that the huTLR4 transgene may not function to their fullest capacity in a mouse background as it does in humans. However, our data from splenic murine macrophages stimulated with Leptospira (Figure 7) clearly shows that the huTLR4 receptor is fully functional in mouse macrophages as they produced as much TNF- $\alpha$ after stimulation with increasing doses of the E. coli LPS TLR4 agonist as did WT mice. Although stimulation of TLR9 and TLR2 led to equivalent production of TNF- $\alpha$ by WT, huTLR4 and muTLR $4^{\text {Lps-del }}$ mouse macrophages, recognition of heat killed Leptospira was decreased in huTLR4 when compared to WT, which is consistent with the lack of sensing of leptospiral-LPS by human TLR4 (1).

When we analyzed proportions of splenic T cells two weeks post infection with $L$. interrogans (Figures 5 and 6) we found increased populations of CD4+ effector T cells in infected versus uninfected mice in WT, huTLR4 and muTLR $4^{\text {Lps-del }}$ which was consistent with our previous observations in $\mathrm{C} 3 \mathrm{H}-\mathrm{HeJ}$ mice (8, 10). Furthermore, the differences observed in CD3+CD8+ T cells suggest that cytotoxic CD8+ $\mathrm{T}$ cells could be engaged in mounting an immune response to Leptospira. Interestingly, in cattle vaccinated with a Leptospira bacterin and subsequently challenged with L. borgpetersenii serovar Hardjo, CD8+ were the only $\mathrm{T}$ cells that proliferated 6 weeks post infection (22). Thus, independently of genetic background, effector CD4+ T cells promote the $\mathrm{T}$ cell response to L. interrogans infection and, quite possibly, CD8+ T cells may be involved.

Regarding differences in infection in immunocompetent (C57BL/6J WT) versus immunocompromised mice (C3H-HeJ, and C57BL/6.10J TLR4 ${ }^{\text {Lps-del }}$ and C57BL/6J TLR4 ${ }^{\mathrm{KO}}$ ), Leptospira persistence in WT mice, shown in ours and other studies (21) fulfills their role as host reservoirs. In the current study we show that Leptospira dissemination, although lower in WT C57BL/6 than in $\mathrm{C} 3 \mathrm{H}-\mathrm{HeJ}$ (8), can be quantified and the immune marker profiles of infection are similar between the two strains. The differences in order of magnitude observed between the two strains simply allow for a more robust quantification of dissemination and inflammation in $\mathrm{C} 3 \mathrm{H}-\mathrm{HeJ}(8,20)$. It is however important to note that Leptospira disseminated and caused pathology in wild-type C57BL/6J which carry a fully functional murine TLR4. This could be explained by the recent finding that leptospiral-LPS also subverts murine TLR4 recognition (23). Indeed, leptospiral-LPS recognition by murine TLR4 is only partial, since only the MyD88 pathway is engaged, not the endosomal TRIF pathway. This avoidance is due to LPS-associated lipoproteins that interfere with CD14 binding of leptospiral-LPS, which block the internalization of TLR4. As a consequence of TRIF avoidance, secretion of type I interferons and nitric oxide production upon Leptospira infection are decreased (23). This somewhat altered TLR4 response could explain the dissemination and kidney colonization after Leptospira infection in mice and it can provide a clue about the lack of profound differences between huTLR4 and WT mice after infection. In addition, Leptospira evade different PRRs, such as TLR5 and NOD proteins, which could contribute to the poor efficiency of macrophages and phagocytes facing Leptospira (24). In this context, the overall role of TLR4 sensing Leptospira could be minimal although its presence would still be important. For example, TLR4 expressed by B cells was shown to be key in early IgM production in mice, which is partially able to counteract Leptospira infection (4). We may speculate that early IgM production, activated three days post infection, may be linked to a role of TLR4, as it was recently shown that B1 cell activation and tumorreactive IgM production were defective in TLR $4^{\mathrm{KO}}$ mice (25).

In conclusion, we expected huTLR4 mice to have been more permissive of Leptospira escape and more susceptible to leptospirosis than congenic wild-type mice. Our hypothesis did not prove to be true. Our data suggests that less recognition of 
leptospiral-LPS by huTLR4 did not affect overall infection and does not result in increased pathology of the kidney. These data indicate that an intact TLR4 gene, be it mouse or human, is necessary to control pathology caused by Leptospira. Unexpectedly, this could help explain why $\sim 90 \%$ of people infected with pathogenic Leptospira (26) are fairly tolerant of this pathogen and do not develop acute Weil's disease.

\section{DATA AVAILABILITY STATEMENT}

The raw data supporting the conclusions of this article will be made available by the authors, without undue reservation.

\section{ETHICS STATEMENT}

The animal study was reviewed and approved by Institutional Animal Care and Use Committee, University of Tennessee Health Science Center (UTHSC).

\section{REFERENCES}

1. Nahori MA, Fournie-Amazouz E, Que-Gewirth NS, Balloy V, Chignard M, Raetz CR, et al. Differential TLR recognition of leptospiral lipid A and lipopolysaccharide in murine and human cells. J Immunol (2005) 175:6022-31. doi: 10.4049/jimmunol.175.9.6022

2. Que-Gewirth NL, Ribeiro AA, Kalb SR, Cotter RJ, Bulach DM, Adler B, et al. A methylated phosphate group and four amide-linked acyl chains in leptospira interrogans lipid A. The membrane anchor of an unusual lipopolysaccharide that activates TLR2. J Biol Chem (2004) 279:25420-9. doi: 10.1074/jbc.M400598200

3. Scior T, Alexander C, Zaehringer U. Reviewing and identifying amino acids of human, murine, canine and equine TLR4 / MD-2 receptor complexes conferring endotoxic innate immunity activation by LPS/lipid A, or antagonistic effects by Eritoran, in contrast to species-dependent modulation by lipid IVa. Comput Struct Biotechnol J (2013) 5:e201302012. doi: 10.5936/csbj.201302012

4. Chassin C, Picardeau M, Goujon JM, Bourhy P, Quellard N, Darche S, et al. TLR4- and TLR2-mediated B cell responses control the clearance of the bacterial pathogen, Leptospira interrogans. J Immunol (2009) 183:2669-77. doi: 10.4049/jimmunol.0900506

5. Pereira MM, Andrade J, Marchevsky RS, Ribeiro dos Santos R. Morphological characterization of lung and kidney lesions in $\mathrm{C} 3 \mathrm{H} / \mathrm{HeJ}$ mice infected with Leptospira interrogans serovar icterohaemorrhagiae: defect of CD4+ and CD8 + T-cells are prognosticators of the disease progression. Exp Toxicol Pathol (1998) 50:191-8. doi: 10.1016/S0940-2993(98)80083-3

6. Nally JE, Fishbein MC, Blanco DR, Lovett MA. Lethal infection of C3H/HeJ and $\mathrm{C} 3 \mathrm{H} / \mathrm{SCID}$ mice with an isolate of Leptospira interrogans serovar copenhageni. Infect Immun (2005) 73:7014-7. doi: 10.1128/IAI.73.10.70147017.2005

7. Viriyakosol S, Matthias MA, Swancutt MA, Kirkland TN, Vinetz JM. Toll-like receptor 4 protects against lethal Leptospira interrogans serovar icterohaemorrhagiae infection and contributes to in vivo control of leptospiral burden. Infect Immun (2006) 74:887-95. doi: 10.1128/IAI.74.2.887-895.2006

8. Richer L, Potula HH, Melo R, Vieira A, Gomes-Solecki M. Mouse model for sublethal Leptospira interrogans infection. Infect Immun (2015) 83:4693-700. doi: 10.1128/IAI.01115-15

9. Vogel SN, Johnson D, Perera PY, Medvedev A, Lariviere L, Qureshi ST, et al. Cutting edge: functional characterization of the effect of the $\mathrm{C} 3 \mathrm{H} / \mathrm{HeJ}$ defect in mice that lack an Lpsn gene: in vivo evidence for a dominant negative mutation. J Immunol (1999) 162(10):5666-70.

\section{AUTHOR CONTRIBUTIONS}

MSG is the corresponding author who conceptualized the project, procured funding, designed experiments, data analysis, prepared figures and wrote the manuscript. NN is the first author who performed the experiments, designed experiments, assisted in writing, data analysis, assisted in preparing figures. MS performed experiments. AMH provided HuTLR/MD2 mice, designed and performed experiments, data analysis and critical review. CW provided critical review and assisted in writing the manuscript. All authors contributed to the article and approved the submitted version.

\section{FUNDING}

We thank the Flow Cytometry and Cell Sorting Core at UTHSC. This work was supported by Public Health Service grants R44AI096551, R43AI136551, R21AI142129 (MG-S) and from the National Institutes of Health and supported by an Institut Pasteur grant PTR66-2017 (CW).

10. Potula HH, Richer L, Werts C, Gomes-Solecki M. Pre-treatment with Lactobacillus plantarum prevents severe pathogenesis in mice infected with Leptospira interrogans and may be associated with recruitment of myeloid cells. PLoS Negl Trop Dis (2017) 11:e0005870. doi: 10.1371/journal.pntd.0005870

11. Sullivan JP, Nair N, Potula HH, Gomes-Solecki M. Eye-Drop Inoculation Leads to Sublethal Leptospirosis in Mice. Infect Immun (2017) 85(4):e0105016. doi: 10.1128/IAI.01050-16

12. Hoshino K, Takeuchi O, Kawai T, Sanjo H, Ogawa T, Takeda Y, et al. Cutting edge: Toll-like receptor 4 (TLR4)-deficient mice are hyporesponsive to lipopolysaccharide: evidence for TLR4 as the Lps gene product. J Immunol (1999) 162(7):3749-52.

13. Hajjar AM, Ernst RK, Fortuno ES3, Brasfield AS, Yam CS, Newlon LA, et al. Humanized TLR4/MD-2 mice reveal LPS recognition differentially impacts susceptibility to Yersinia pestis and Salmonella enterica. PloS Pathog (2012) 8: e1002963. doi: 10.1371/journal.ppat.1002963

14. Nagai Y, Akashi S, Nagafuku M, Ogata M, Iwakura Y, Akira S, et al. Essential role of MD-2 in LPS responsiveness and TLR4 distribution. Nat Immunol (2002) 3:667-72. doi: 10.1038/ni809

15. Vogel SN, Hansen CT, Rosenstreich DL. Characterization of a congenitally LPS-resistant, athymic mouse strain. J Immunol (1979) 122(2):619-22.

16. Poltorak A, He X, Smirnova I, Liu MY, Van Huffel C, Du X, et al. Defective LPS signaling in $\mathrm{C} 3 \mathrm{H} / \mathrm{HeJ}$ and $\mathrm{C} 57 \mathrm{BL} / 10 \mathrm{ScCr}$ mice: mutations in Tlr4 gene. Science (1998) 282:2085-8. doi: 10.1126/science.282.5396.2085

17. Nair N, Gomes-Solecki M. A Mouse Model of Sublethal Leptospirosis: Protocols for Infection with Leptospira Through Natural Transmission Routes, for Monitoring Clinical and Molecular Scores of Disease, and for Evaluation of the Host Immune Response. Curr Protoc Microbiol (2020) 59: e127. doi: 10.1002/cpmc.127

18. Hajjar AM, Ernst RK, Yi J, Yam CS, Miller SI. Expression level of human TLR4 rather than sequence is the key determinant of LPS responsiveness. PLoS One (2017) 12:e0186308. doi: 10.1371/journal.pone.0186308

19. Werts C, Tapping RI, Mathison JC, Chuang TH, Kravchenko V, Saint Girons I, et al. Leptospiral lipopolysaccharide activates cells through a TLR2dependent mechanism. Nat Immunol (2001) 2:346-52. doi: 10.1038/86354

20. Nair N, Guedes MS, Werts C, Gomes-Solecki M. The route of infection with Leptospira interrogans serovar Copenhageni affects the kinetics of bacterial dissemination and kidney colonization. PLoS Negl Trop Dis (2020) 14: e0007950. doi: 10.1371/journal.pntd.0007950

21. Fanton d'Andon M, Quellard N, Fernandez B, Ratet G, Lacroix-Lamande S, Vandewalle A, et al. Leptospira Interrogans induces fibrosis in the mouse 
kidney through Inos-dependent, TLR- and NLR-independent signaling pathways. PLoS Negl Trop Dis (2014) 8:e2664. doi: 10.1371/journal. pntd.0002664

22. Zuerner RL, Alt DP, Palmer MV, Thacker TC, Olsen SC. A Leptospira borgpetersenii serovar Hardjo vaccine induces a Th1 response, activates NK cells, and reduces renal colonization. Clin Vaccine Immunol (2011) 18:684-91. doi: 10.1128/CVI.00288-10

23. Bonhomme D, Santecchia I, Vernel-Pauillac F, Caroff M, Germon P, Murray G, et al. Leptospiral LPS escapes mouse TLR4 internalization and TRIFassociated antimicrobial responses through $\mathrm{O}$ antigen and associated lipoproteins. PLoS Pathog (2020) 16:e1008639. doi: 10.1371/journal. ppat.1008639

24. Santecchia I, Ferrer MF, Vieira ML, Gomez RM, Werts C. Phagocyte Escape of Leptospira: The Role of TLRs and NLRs. Front Immunol (2020) 11:571816. doi: 10.3389/fimmu.2020.571816

25. Dyevoich AM, Disher NS, Haro MA, Haas KM. A TLR4-TRIF-dependent signaling pathway is required for protective natural tumor-reactive IgM production by B1 cells. Cancer Immunol Immunother (2020) 69:2113-24. doi: 10.1007/s00262-020-02607-7

26. Costa F, Hagan JE, Calcagno J, Kane M, Torgerson P, Martinez-Silveira MS, et al. Global Morbidity and Mortality of Leptospirosis: A Systematic Review. PLoS Negl Trop Dis (2015) 9:e0003898. doi: 10.1371/journal.pntd.0003898

Conflict of Interest: The authors declare that the research was conducted in the absence of any commercial or financial relationships that could be construed as a potential conflict of interest.

Copyright (c) 2021 Nair, Guedes, Hajjar, Werts and Gomes-Solecki. This is an openaccess article distributed under the terms of the Creative Commons Attribution License (CC BY). The use, distribution or reproduction in other forums is permitted, provided the original author(s) and the copyright owner(s) are credited and that the original publication in this journal is cited, in accordance with accepted academic practice. No use, distribution or reproduction is permitted which does not comply with these terms. 